\title{
Magnetic Resonance Imaging Verification of a Case of Sacrococcygeal Teratoma
}

\author{
Kreshnike Dedushi ${ }^{\mathrm{a}, \mathrm{b}, \mathrm{c}}$, Serbeze Kabashi ${ }^{\mathrm{a}, \mathrm{b}}$, f, Sefedin Mucaja, d, Naser Ramadani ${ }^{\mathrm{a}, \mathrm{d}}$, \\ Astrit Hoxhaje, Jeton Shatri $^{\mathrm{a}, \mathrm{b}}$, Gazmend Hasbahta ${ }^{\mathrm{b}}$
}

\begin{abstract}
Although rare, sacrococcygeal teratoma is the most common congenital neoplasm, occurring in 1 in 40,000 infants. Approximately $75 \%$ of affected infants are female. The aim of the present study was to correlate ultrasonography and magnetic resonance imaging (MRI) findings in patients with fetal sacrococcygeal teratoma. Three pregnant women in 27th week of gestation underwent fetal MRI after ultrasonography examination, with findings suggestive for fetal sacrococcygeal teratoma. Tumor size, location, extent and content were evaluated both by MRI and ultrasonography. Findings regarding tumor location, size and content were similar for both methods. There was a large wellcircumscribed mixed, cystic/solid oval mass, originating from right sacro-gluteal region and projecting into the amniotic cavity, $132 \times$ $110 \times 76 \mathrm{~mm}$ in size. The mass had a heterogeneous appearance. The T1 high signal suggested fat component of the tumor, while T1 and T2 hypointense components suggested calcified/bony components. There was also T1 hypointense component consistent with cystic and fluid component. The imaging findings were characteristic for sacrococcygeal teratoma. There was not obvious lumbar or thoracic spinal involvement. There was no gross intrapelvic or abdominal extension, and even sacrum and coccyx appeared deformed. The amount of amniotic fluid was increased. MRI was superior to ultrasonography in the evaluation of the exact tumor extent, accurately demonstrating pelvic involvement in all of the three cases. Fetal MRI has shown to be a valuable adjunct to obstetric sonography in the evaluation of fetal sacrococcygeal teratoma, because of its higher accuracy in the determination of tumors extent and content, playing a significant role in the therapeutic planning and increasing the chances of cure for these fetuses.
\end{abstract}

Manuscript accepted for publication May 18, 2016

${ }^{a}$ Faculty of Medicine, Pristine University, Pristine City, 10000, Kosovo bDepartment of Radiology, Diagnostic Centre, UCCK, Pristine City, 10000, Kosovo

'Diagnostic Center, International Health Center "IHC", Pristine City, 10000, Kosovo

${ }^{\mathrm{d}}$ National Institute of Public Health of Kosovo, Pristine City, 10000, Kosovo eAmerican Hospital, Tirana City, 10004, Albania

${ }^{f}$ Corresponding Author: Serbeze Kabashi, Faculty of Medicine, Pristine University; Department of Radiology, Diagnostic Centre, UCCK, Pristine City, 10000, Kosovo.Email: sebikabashi@gmail.com

doi: http://dx.doi.org/10.14740/wjon965w
Keywords: Fetus; Sacrococcygeal teratoma; Ultrasonography; Magnetic resonance imaging

\section{Introduction}

Although rare, sacrococcygeal teratoma is the most common congenital neoplasm, occurring in 1 in 40,000 infants. Approximately $75 \%$ of affected infants are female [1,2]. It has been defined as either a neoplasm composed of tissues from all three germ layers or a neoplasm formed from multiple tissues foreign to the part and lacking organ specificity [3]. The tumor is derived from the pluri-potential cell line originating in Hensen's node and contains components arising from all three germ layers. The perinatal mortality and morbidity rates are high because of high output cardiac failure, preterm delivery, anemia, dystocia, and tumor rupture. The intrapelvic and intraabdominal extent of the sacrococcygeal teratoma and its compressive effect on the urinary tract also affect perinatal morbidity; these elements may prolong neonatal surgery and increase the risk of preoperative hemorrhage. Prenatal assessment of the fetus is critical for counseling the parents and planning surgical options. Also, with the development of in utero treatment for sacrococcygeal teratoma $[4,5]$, magnetic resonance imaging (MRI) offers superior anatomic resolution, regardless of fetal orientation, and provides an image display that is more intuitively comprehensible to the patient and to many consulting physicians [6].

We have described the clinical findings, diagnostic procedures and management of a fetus in 27th gestational week, in a 24-year-old pregnant patient. She was referred by gynecologist for fetal MRI examination since ultrasonography findings suggested possible fetal sacrococcygeal teratoma. It is an extremely rare case of the very few reported in the published works and, to the best of our knowledge, it is the second one.

\section{Case Report}

A 24-year-old female patient, first time pregnant, was referred by gynecologist for fetal MRI after ultrasonography examination that showed a mixed, solid/cystic tumorous oval mass in the sacral region of the embryo (Fig. 1a, b). The patient visited 

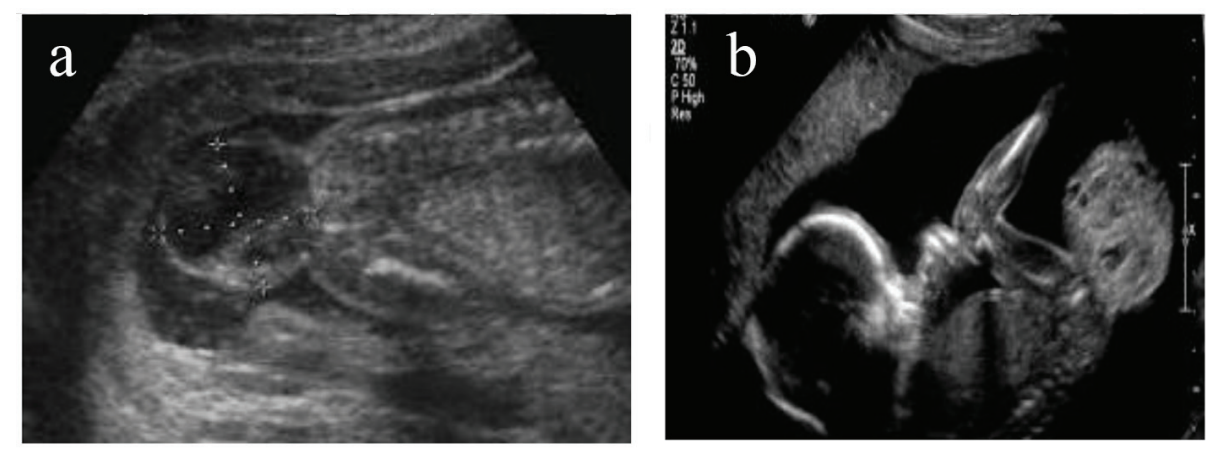

Figure 1. $(a, b)$ Ultrasonography examination that showed mixed, solid/cystic tumorous oval mass in the sacral region of the embryo.

our Diagnostic Center "IHC" and accomplished fetal MRI. The technical protocol applied was suitable for embryo-fetal imaging. Multiplanar and multisequential MRI of the fetus was performed without contrast, with attention to the fetal CNS and the result showed a large well-circumscribed mixed, cystic/solid oval mass, originating from the right sacro-gluteal region and projecting into the amniotic cavity, $132 \times 110 \times 76$ $\mathrm{mm}$ in size. The mass has a heterogeneous appearance.

The T1 high signal suggested fat component of the tumor, while T1 and T2 hypointense components suggested calcified/ bony components. There was also $\mathrm{T} 1$ hypointense component consistent with cystic and fluid component. The imaging findings were characteristic for sacrococcygeal teratoma.

There was no obvious lumbar or thoracic spinal involvement. There was no gross intrapelvic or abdominal extension, and even sacrum and coccyx appeared deformed (Fig. 2a-e). The cervix was long at $40 \mathrm{~mm}$ and closed. The placenta was high and anterior. There was a normal three vessel cord without evident loops or knots. The amount of amniotic fluid was prominent. There was a single live intrauterine gestation. Current fetal lie was vertex, with spine to the mother's left. At the level of the fetal brain, the degree of gyration and sulcation was as expected for the stated gestational age. No structural abnormalities were noted in the cerebral hemispheres. On T1weighted images, myelination was as expected. The corpus callosum was present and normally formed. The brainstem and cerebellum including vermis had a normal appearance with transverse cerebellar diameter of $31 \mathrm{~mm}$, in the expected range for gestational age. The craniocervical junction was unremarkable with no sign of Chiari or other malformation (Fig. 2f). The ocular globes had a normal and symmetrical appearance. The fetal face showed no midline cleft or other abnormality. The fetal spine was unremarkable. There was no sign of scoliosis or open neural tube defect. The fetal chest and abdomen showed visceral situs solidus with all the developed organs demonstrating proportional size and expected signal intensity on all sequences. Specifically, stomach, gallbladder, kidneys and urinary bladder were well seen and normal in appearance.

\section{Discussion}

Fetal sacrococcygeal teratomas diagnosed in utero carry a high risk of preterm delivery (50\%), a mortality rate of $15-35 \%$, and a morbidity rate of $12-68 \%$. Prognosis seems to be related not to the size of the mass but rather to its content and extent. Solid hypervascularized masses carry a poorer prognosis than purely cystic masses $[7,8]$. Fetal hydrops, hemorrhage, or rupture of the sacrococcygeal teratoma are the main complications associated with a high mortality rate. The degree of intrapelvic or abdominal extent of the mass affects the morbidity rate related to postnatal surgery by increasing the duration of surgery and the risk of hemorrhage. Compression of the fetal urinary tract may result in urologic complications ( $42 \%$ in one series) [9]. Therefore, it is important to evaluate these tumors as precisely as possible. There has been a rapid increase in the use of MRI for the assessment of the normal and abnormal anatomy of the fetus $[10,11]$. Results clearly show that MRI is superior to sonography in assessing the intrapelvic and intraspinal extent of tumor and in discerning the presence and physiologic effects of compression of pelvic organs by the tumor. These advantages allow more accurate prognostic counseling for patients presenting with sacrococcygeal teratoma and improve pre- and perinatal management by providing accurate data for decisions regarding fetal surgery, tumor decompression, and timing of delivery. The advantage of MRI is primarily related to the absence of acoustic shadowing by the fetal pelvic bones that interferes with sonography visualization. MRI also enhanced the assessment of the content of sacrococcygeal teratoma. Fetal sacrococcygeal teratoma may be cystic, solid, or mixed in sonographic appearance and may contain characteristic echogenic patterns secondary to areas of tumor necrosis, cystic degeneration, internal hemorrhage, and calcification. The prognosis of prenatally detected sacrococcygeal teratoma seems to be related not only to the size of the mass but also to its content.

Fetuses with large echogenic tumors on MRI need frequent monitoring using sonography and echocardiography for evolution of high cardiac output physiology to determine the need for intervention [12]. Fetuses with predominantly solid and highly vascularized masses have a poorer prognosis than fetuses with tumors that are mainly cystic and avascular in appearance. Surgeons at our institution found that MRI helped them mentally visualize the content and extent of the sacrococcygeal teratoma before delivery. In most cases, neonatal surgery is required soon after cesarean delivery, and the anatomic details of tumor extent and involvement of adjacent structures 

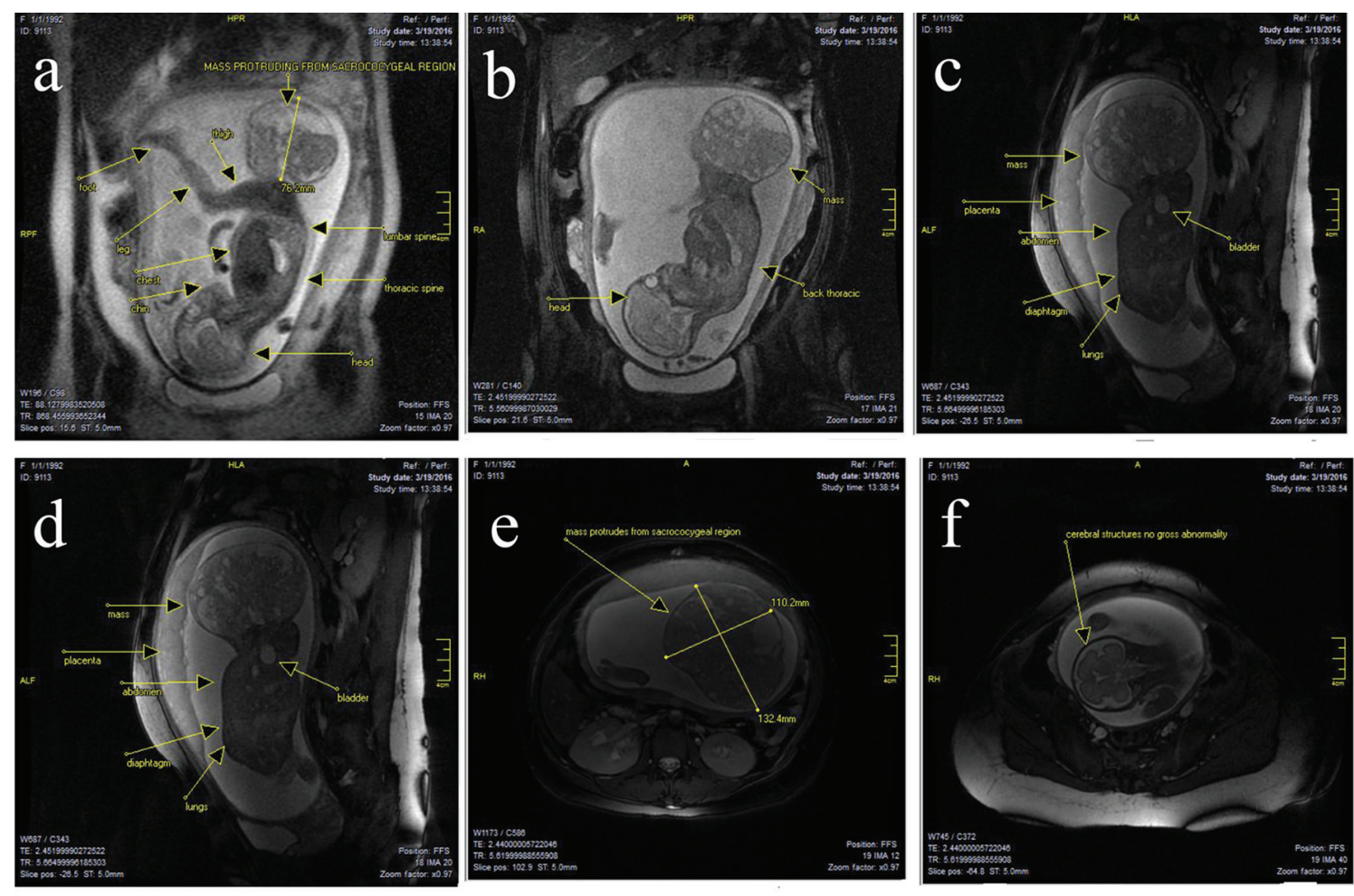

Figure 2. (a-f) There was a large well-circumscribed mixed cystic solid oval mass, originating from right sacro-gluteal region and projecting into the amniotic cavity, $132 \times 110 \times 76 \mathrm{~mm}$ in size. The mass had a heterogenous appearance. There were T1 high signal suggesting fat component and there are T1 and T2 hypointense components suggesting calcific bony components. There was also T1 hypointense components consistent with cystic and fluid component. Findings suggested sacrococcygeal teratoma. There was no obvious lumbar or thoracic spinal involvement. There was no gross intrapelvic or abdominal extension. But sacrum and coccyx appeared deformed. At the level of the fetal brain, the degree of gyration and sulcation was as expected for the stated gestational age. No structural abnormalities were noted in the cerebral hemispheres. On T1-weighted images, myelination was as expected. The corpus callosum was present and normally formed. The brainstem and cerebellum including vermis had a normal appearance with transverse cerebellar diameter of $31 \mathrm{~mm}$, in the expected range for gestational age. The craniocervical junction was unremarkable with no sign of Chiari or other malformation.

may affect the surgical approach. No postnatal tumor imaging was done before surgery. In two fetuses, extension into the spinal canal was visible only on MRI. Patients with significant intrapelvic extension of the tumor may need a combined abdominoperineal approach to control the blood supply and achieve complete surgical resection. The colon is well visualized and distinguished from an intraabdominal sacrococcygeal teratoma because the meconium has high signal intensity on T1-weighted images, providing valuable information about atypical colon deviation or involvement before surgery [13].

\section{Conclusion}

In conclusion, MRI provides additional and helpful information for the assessment of fetal sacrococcygeal teratoma. MRI is a proven, established imaging modality for evaluating fetal anomalies that are not well or completely assessed by sonography. MRI is used for problem solving and only in select circumstances for screening. Properly performed and interpreted, MRI not only contributes to diagnosis but also serves as an important guide to treatment, delivery planning and coun- seling. However, ultrasonography is the screening modality of choice in the fetus. Fetal MRI should be performed only for a valid medical reason and only after careful consideration of ultrasonographic findings or family history of abnormality for which screening with MRI might be beneficial.

Based on our experience using prenatal MRI for evaluation of fetal sacrococcygeal teratoma, we recommend that all fetuses with appearance of sacrococcygeal teratoma on ultrasonography undergo MRI evaluation to assess exact tumor size, content, and intraabdominal extent to optimize pre-, periand postnatal management.

\section{Conflicts of Interest}

The authors have no conflicts of interest to declare.

\section{Author Contributions}

All authors of this paper have contributed in all phases. Final proof reading was made by the first author. All authors have 
read and approved the final manuscript.

\section{References}

1. Chisholm CA, Heider AL, Kuller JA, von Allmen D, McMahon MJ, Chescheir NC. Prenatal diagnosis and perinatal management of fetal sacrococcygeal teratoma. Am J Perinatol. 1999;16(1):47-50.

2. Brace V, Grant SR, Brackley KJ, Kilby MD, Whittle MJ. Prenatal diagnosis and outcome in sacrococcygeal teratomas: a review of cases between 1992 and 1998. Prenat Diagn. 2000;20(1):51-55.

3. Flake AW. The fetus with sacrococcygealteratoma. In: Harrison MR, Evans MI, Adzick NS, HolzgreveW, Eds. The unborn patient, 3rd ed. Philadelphia PA: WB Saunders, 2000:315-323.

4. Adzick NS, Crombleholme TM, Morgan MA, Quinn TM. A rapidly growing fetal teratoma. Lancet. 1997;349(9051):538.

5. Paek BW, Jennings RW, Harrison MR, Filly RA, Tacy TA, Farmer DL, Albanese CT. Radiofrequency ablation of human fetal sacrococcygeal teratoma. Am J Obstet Gynecol. 2001;184(3):503-507.

6. Enrico Danzer, Anne M. Hubbard, Holly L. Hedrick, Mark P. Johnson, R. Douglas Wilson, Lori J. Howell, Alan W. Flake, N. Scott Adzick. Diagnosis and Characterization of Fetal SacrococcygealTeratoma with Prenatal MRI. Ameri- can Journal of Roentgenology. 2006;187(4):350-356.

7. Holterman AX, Filiatrault D, Lallier M, Youssef S. The natural history of sacrococcygeal teratomas diagnosed through routine obstetric sonogram: a single institution experience. J Pediatr Surg. 1998;33(6):899-903.

8. Chuileannain FN, Woodrow N, de Crespigny L. Prenatal diagnosis and management of sacrococcygeal teratoma. Aust N Z J Obstet Gynaecol. 1999;39(4):497-501.

9. Westerburg B, Feldstein VA, Sandberg PL, Lopoo JB, Harrison MR, Albanese CT. Sonographic prognostic factors in fetuses with sacrococcygeal teratoma. J Pediatr Surg. 2000;35(2):322-325; discussion 325-326.

10. Amin RS, Nikolaidis P, Kawashima A, Kramer LA, Ernst RD. Normal anatomy of the fetus at MR imaging. Radiographics. 1999;19(Spec No):S201-214.

11. Shinmoto H, Kashima K, Yuasa Y, Tanimoto A, Morikawa Y, Ishimoto H, Yoshimura Y, et al. MR imaging of non-CNS fetal abnormalities: a pictorial essay. Radiographics. 2000;20(5):1227-1243.

12. Hedrick HL, Flake AW, Crombleholme TM, Howell LJ, Johnson MP, Wilson RD, Adzick NS. Sacrococcygeal teratoma: prenatal assessment, fetal intervention, and outcome. J Pediatr Surg. 2004;39(3):430-438; discussion 430-438.

13. Avni FE, Guibaud L, Robert Y, Segers V, Ziereisen F, Delaet MH, Metens T. MR imaging of fetal sacrococcygeal teratoma: diagnosis and assessment. AJR Am J Roentgenol. 2002;178(1):179-183. 